\title{
Trichostasis spinulosa of the scalp mimicking Alopecia Areata black dots
}

\author{
Flavianne Sobral Cardoso Chagas ${ }^{1,2}$ \\ Isabella Ibrahim Doche Soares ${ }^{2}$ \\ Ricardo Romiti ${ }^{2}$
}

Aline Donati ${ }^{2}$

Neusa Sakai Valente ${ }^{2}$

DOI: http://dx.doi.org/10.1590/abd1806-4841.20142407

\begin{abstract}
Alopecia areata is a common autoimmune disorder that leads to nonscarring hair loss. Black dots, also called comedo-like cadaver hairs, can be found in almost $50 \%$ of alopecia areata patients and indicate disease activity. Trichostasis spinulosa is a follicular disorder resulting from the retention of numerous hairs surrounded by a keratinous sheath in dilated follicles. Trichostasis spinulosa is a relatively common but underdiagnosed disorder of hair follicles. Here, we describe a man with alopecia areata of the eyebrows, androgenetic alopecia and trichostasis spinulosa at the vertex and show how dermoscopy can be useful in distinguishing black dots from Trichostasis spinulosa lesions.
\end{abstract}

Keywords: Alopecia; Alopecia areata; Dermoscopy; Hair diseases

Alopecia areata (AA) is a chronic autoimmune disease targeting hair follicles. The disorder is usually characterized by limited alopecic patches on the scalp, but more severe forms may affect the entire scalp (alopecia totalis) or body (alopecia universalis). ${ }^{1}$ Recently, dermoscopy has become an important tool for the diagnosis of AA, especially in cases with unusual presentations. Dermoscopy findings most associated with AA are: black dots (also called comedo-like cadaver hairs), tapering hairs corresponding to "exclamation mark hairs", broken hairs, yellow dots and short regrowing vellus hairs.,3,4

Trichostasis spinulosa (TS) is a follicular disorder resulting from the retention of numerous hairs surrounded by a keratinous sheath in a dilated follicle. It most often presents as an isolated finding, but it can also be detected as secondary to expansile nondestructive lesions that narrow hair infundibulae, such as melanocytic nevi, seborrheic keratoses, syringomas, or nodular basal cell carcinomas ${ }^{5}$, or more rarely, as the result of folliculosebaceous hamartomas with multiple units around a central pore (trichofolliculomas). In any case, retained hair shafts are kept together inside follicular openings by various degrees of infundibular keratosis. Dermoscopy enables better observation of multiple vellus hairs erupting through the follicles and is useful for TS diagnosis. ${ }^{6}$
A 56-year-old Brazilian man presented with a 6month history of eyebrow loss. He referred typical male pattern alopecia since the age of 20 and denied any recent change in scalp alopecia. His family history was positive for androgenetic alopecia, but there was no personal or familial history of alopecia areata. The patient denied any comorbidity and did not take any regular medication.

Upon physical examination, eyebrows were completely absent and eyelashes were sparse. The scalp showed frontal, vertex, and parietal hair thinning with decreased hair density. There were multiple follicular dark structures over the vertex. They were pinheadsized and clinically resembled AA black dots (Figure 1A). The pull test was negative over the scalp and there were no other areas of alopecia over the body.

Dermoscopy of the vertex area of the scalp revealed black dot-like structures, which were indeed tufts of light pigmented vellus hairs, tightly held together inside one follicular opening (Figure 1B). Slight compression, used for immersion dermoscopy, spread the group of hairs into different lengths and directions, facilitating visualization of individual hair shaft tips just above the epidermal level.

Increased proportion of thin hairs, reduced hair density and visible honeycomb pattern were also visible on the vertex area, and compatible with concur-

Received on 07.01.2013.

Approved by the Advisory Board and accepted for publication on 26.06.2013.

Work performed at the Departamento de Dermatologia do Hospital das Clínicas da Faculdade de Medicina da Universidade de São Paulo (HC-FMUSP) - São Paulo (SP), Brazil.

Conflict of interest: None

Financial funding: None

Universidade Federal de Sergipe (UFS) - Aracaju (SE), Brazil

Universidade de São Paulo (USP) - São Paulo (SP), Brazil.

(C2014 by Anais Brasileiros de Dermatologia 

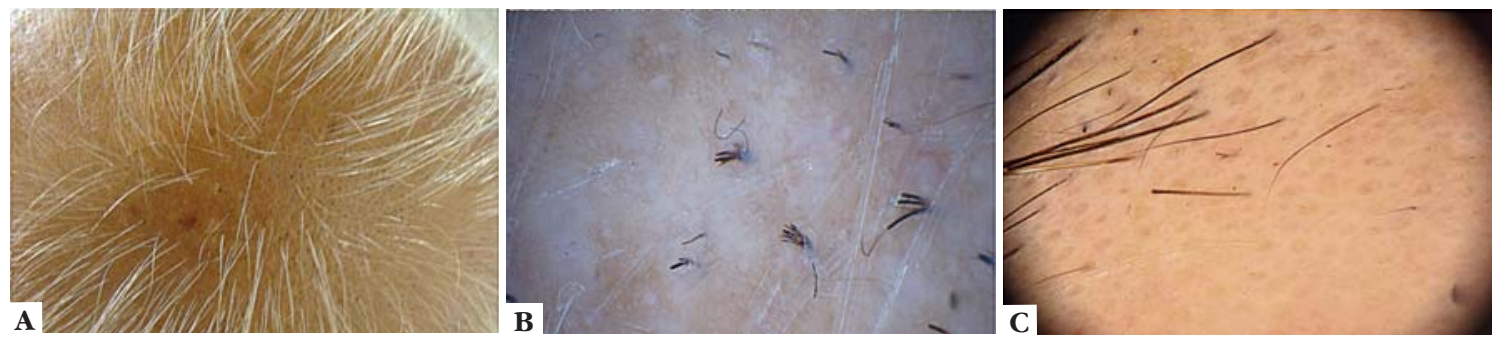

FigURE 1: A. Clinical aspect of the vertex area: black dots were visualized inside an alopecia area containing a decreased density of white terminal hairs; B. Dermoscopy of vertex area showed tufts of tiny black hairs emerging from follicular ostia. (Immersion dermoscopy, 20x augmentation lens attached to a Sony digital camera); C. Dermoscopy of eyebrows showed comedo-like cadaver hairs in an area affected by alopecia areata

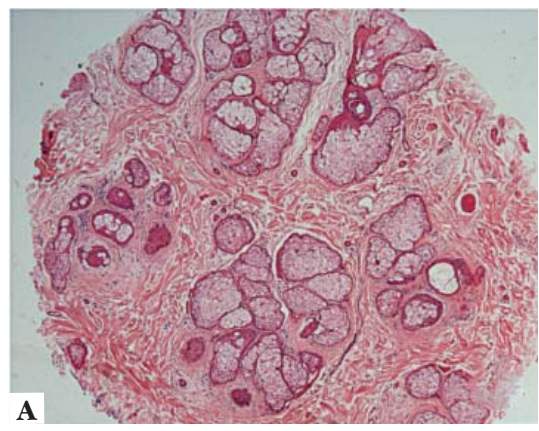

rent androgenetic alopecia. Dermoscopy of the eyebrows showed yellow dots and true comedo-like cadaver hairs (black dots), confirming the diagnosis of alopecia areata (Figure 1C).

Laboratory tests were performed in order to screen for other autoimmune disorders; all were within normal limits.

The patient agreed to a biopsy performed on his scalp area, but not the eyebrows. Histopathology of the scalp was characteristic of androgenetic alopecia and showed an inverted proportion of vellus: terminal follicles with preserved sebaceous glands (Figure 2A). Most superficial levels of horizontal slides depicted groups of 3-5 thin hair shafts inside dilated infundibuli, confirming the diagnosis of trichostasis spinulosa (Figure 2B).

Diagnosis of androgenetic alopecia Hamilton III $\mathrm{V}$ associated with trichostasis spinulosa lesions was made for the scalp alopecia, but no treatment was initiated because the patient did not complain about it. Clinical and dermoscopic diagnosis of alopecia areata of the eyebrows was done and intralesional injection of triamcinolone $2.5 \mathrm{mg} / \mathrm{ml}$ every 6 weeks was started with partial response, but frequent relapse after discontinuation of treatment.

The use of dermoscopy in trichology has proved useful for many disorders. ${ }^{7}$ In AA, "exclamation mark" hairs (also called tapering hairs) are an important diagnostic clue that can be better visualized via dermoscopy. Broken hairs and black dots have also good specificity for the diagnosis of AA and correlate positively with disease activity. ${ }^{2}$

Black dots can be found in almost $50 \%$ of AA cases. ${ }^{4}$ The mechanism underlying black dots formation involves the sudden arrest of the hair cycle caused by the autoimmune mediated inflammatory process affecting the bulb region of anagen follicles. ${ }^{8}$ Terminal pigmented shafts of inflamed follicles suddenly become weak and thin, leading to breakage at different levels, with posterior dissolution of the shaft into an amorphous pigmented material inside the follicular opening, which appears clinically as black dots.

Black dots can also be seen in other clinical settings, presenting with hair breakage such as trichotillomania, traction alopecia, and tinea capitis. Clinical aspects of trichostasis spinulosa can be misinterpreted as AA black dots but dermoscopy can easily distinguish it, revealing the classic hair tufts instead of the amorphous pigmented material inside hair openings.

We presented the case of a patient who had alopecia areata in the eyebrows and trichostasis spinulosa, plus androgenetic alopecia on the scalp. Dermoscopy was useful in diagnosing correctly both disorders.

Dermoscopy is a practical, simple and noninvasive method that helps dermatologists diagnose many hair disorders in routine practice. It helps avoid more invasive procedures, improves diagnostic accuracy and allows prompt introduction of appropriate therapeutic measures. $\square$ 


\section{REFERENCES}

1. Alkhalifah A, Alsantali A, Wang E, McElwee KJ, Shapiro J. Alopecia areata update : part I. Clinical picture, histopathology, and pathogenesis. J Am Acad Dermatol. 2010;62:177-88

2. Inui S, Nakajima T, Nakagawa K, Itami S. Clinical significance of dermoscopy in alopecia areata: analysis of 300 cases. Int J Dermatol. 2008;47:688-93.

3. Tosti A, Whiting D, lorizzo M, Pazzaglia M, Misciali C, Vincenzi C, et al. The role of scalp dermoscopy in the diagnosis of alopecia areata incognita. J Am Acad Dermatol. 2008;59:64-7.

4. Ross EK, Vincenzi C, Tosti A. Videodermoscopy in the evaluation of hair and scalp disorders. J Am Acad Dermatol. 2006;55:799-806.

5. Navarini AA, Ziegler M, Kolm I, Weibel L, Huber C, Trüeb RM. Trueb RM. MinoxidilInduced Trichostasis Spinulosa of Terminal Hair. Arch Dermatol. 2010;146:1434-5.

6. Pozo L, Bowling J, Perrett CM, Bull R, Diaz-Cano SJ. Dermoscopy of trichostasis spinulosa. Arch Dermatol. 2008:144:1088.

7. Tosti A, Duque-Estrada B. Dermoscopy in hair disorders. J Egypt Women Dermatol Soc. 2010;7:1-4

8. Torres FN, Azulay L, Abraham LS. Dermoscopic clues to distinguish trichotillomania from patchy alopecia areata. An Bras Dermatol. 2010;85:723-6.
MAILING ADDRESS:

Flavianne Sobral Cardoso Chagas

Av. Dr. Eneas de Carvalho Aguiar, 255 - $3^{\circ}$ Andar

Cerqueira César

05403900 - São Paulo - SP

Brazil

E-mail: flavianne.cardoso@globo.com

How to cite this article: Chagas FSC, Donati A, Soares IID, Valente NS, Romiti R. Trichostasis spinulosa of the scalp mimicking Alopecia Areata black dots. An Bras Dermatol. 2014;89(4):685-7. 